\title{
Improving Participation in Colorectal Cancer Screening: a Randomised Controlled Trial of Sequential Offers of Faecal then Blood Based Non-Invasive Tests
}

\author{
Erin L Symonds ${ }^{1,2 *}$, Susanne Pedersen ${ }^{3}$, Stephen R Cole ${ }^{1,2}$, Joseph Massolino ${ }^{4}$, \\ Daniel Byrne ${ }^{5}$, John Guy ${ }^{6}$, Patricia Backhouse ${ }^{4}$, Robert J Fraser ${ }^{1,7}$, Lawrence \\ LaPointe $^{3}$, Graeme P Young ${ }^{1}$
}

\begin{abstract}
Background: Poor participation rates are often observed in colorectal cancer (CRC) screening programs utilising faecal occult blood tests. This may be from dislike of faecal sampling, or having benign bleeding conditions that can interfere with test results. These barriers may be circumvented by offering a blood-based DNA test for screening. The aim was to determine if program participation could be increased by offering a blood test following faecal immunochemical test (FIT) non-participation. Materials and Methods: People were invited into a CRC screening study through their General Practice and randomised into control or intervention (n=600/group). Both groups were mailed a FIT (matching conventional screening programs). Participation was defined as FIT completion within $12 \mathrm{wk}$. Intervention group non-participants were offered a screening blood test (methylated BCAT1/IKZF1). Overall participation was compared between the groups. Results: After 12wk, FIT participation was $82 \%$ and $81 \%$ in the control and intervention groups. In the intervention 96 FIT nonparticipants were offered the blood test $\mathbf{- 2 2}$ completed this test and 19 completed the FIT instead. Total screening in the intervention group was greater than the control $(88 \%$ vs $82 \%, p<0.01)$. Of 12 invitees who indicated that FIT was inappropriate for them (mainly due to bleeding conditions), 10 completed the blood test $(83 \%)$. Conclusions: Offering a blood test to FIT non-participants increased overall screening participation compared to a conventional FIT program. Blood test participation was particularly high in invitees who considered FIT to be inappropriate for them. A blood test may be a useful adjunct test within a FIT program.
\end{abstract}

Keywords: Colorectal cancer - screening - faecal immunochemical test - methylated blood test - participation - FOBT

Asian Pac J Cancer Prev, 16 (18), 8455-8460

\section{Introduction}

Colorectal cancer (CRC) is one of the most prevalent cancers, with approximately 1.4 million new cases diagnosed and 608,000 deaths worldwide per year (AIHW, 2014). Despite high likelihood of cure when diagnosed at an early stage, CRC remains the second leading cause of death from cancer. To enable early detection, many countries have introduced population-based CRC screening programs, incorporating either guaiac faecal occult blood tests (gFOBT), or the newer immunochemical faecal tests (FIT). We have shown that screening program participation with FIT detects CRC at an earlier and more treatable stage, compared to cancer detected outside of the program (Cole et al., 2013), with other studies supporting a mortality reduction with FOBT use (Mandel et al., 1993; Hardcastle et al., 1996; Zorzi et al., 2014). Despite these proven benefits, current participation in screening programs is modest (AIHW, 2014; Bresalier et al., 2015). If screening is to achieve its full potential as a public health strategy, participation in screening needs to be increased.

Much research has explored demographic and program factors with participation in FOBT-based screening, with a view to identifying and removing barriers to screening. Poor participation is associated with factors such as fatalism (Greiner et al., 2005; Miles et al., 2011), beliefs about CRC prevention and the benefit of screening (Cole et al., 2011); and test technology variables (Cole et al., 2003; Cole et al., 2011; Cole et al., 2012). Dislike of sampling mode is a common reason for non-participation in screening (Worthley et al., 2006), with one study finding that faecal-sample based were the least preferred of various testing options such as colonoscopy, barium enema, sigmoidoscopy and virtual colonoscopy (Marshall 
et al., 2007). Another barrier to participation for some is presented by the biological basis of FOBT. As the test depends on bleeding from neoplasia, any other condition that causes bleeding can also trigger a positive test. Thus, to avoid the high likelihood of false positive FIT results and unnecessary colonoscopy, screening with faecal testing may be contraindicated for people with benign bleeding conditions, such as inflammatory bowel disease, haemorrhoids and radiation proctitis. These problems inherent in faecal testing might be overcome by inclusion of a different type of test within CRC screening programs, such as a blood test.

Next generation molecular diagnostic assays now offer the potential to detect tumour-derived DNA in blood, for example by measuring the level of aberrant methylation in specific genes (deVos et al., 2009). We have shown that the genes BCAT1 and IKZF1 are methylated with high frequency in colorectal neoplastic tissues (Mitchell et al., 2014) and that these methylated genes can be detected in the plasma of patients with CRC (Pedersen et al., 2015a; Pedersen et al., 2015b). The methylated BCAT1/IKZF1 blood test has comparable sensitivity to FIT for CRC, and better specificity (Symonds et al., 2015). These new assays might provide a means to significantly improve participation rates with screening by providing people who cannot or will not screen with a faecal sample-based test with the opportunity to conveniently screen using a blood test. The blood test may be deployed as a second line rescue strategy to engage those who reject the existing screening methods. While it has been suggested that a blood test would be more acceptable and circumvent some of the barriers with other screening methods, participation rates in FOBT non-participants has not been assessed, and this is critical prior to inclusion of any new test within a screening program. The aim of this study was to therefore determine if CRC screening participation could be increased by inclusion of a blood test within a FIT-based screening program.

\section{Materials and Methods}

\section{Study population}

People from three participating primary care practices located within the South Australian metropolitan area (East Adelaide Healthcare, Marden; Chandlers Hill Surgery, Happy Valley; Flagstaff Hill Medical Centre, Flagstaff Hill) were invited to participate in a CRC prevention research study. Invited people $(n=4522)$ were aged 50-74 years (inclusive), were without any major illnesses and were deemed suitable for CRC screening by their practitioner. Following study invitation, 1212 $(27 \%)$ of invitees returned informed consent. The first 1200 consenting individuals were enrolled and randomised into control and intervention groups $(n=600$ per group, Figure 1. Sample size calculated to provide $80 \%$ power to detect a $10 \%$ difference in participation). Randomisation was done via a random number generator, but ensured that co-habiting enrolees were randomised to the same group. Invitees were not informed about the precise nature of the research until they received a mailed FIT screening kit. Ethical approval was obtained from the Southern Adelaide
Clinical Human Research Ethics Committee and the trial is registered at Australian and New Zealand Clinical Trials Registry (trial \#ACTRN12613001365752).

\section{Control group - standard FIT program}

Starting November 2013 all study participants were sent a quantitative FIT kit (OC Sensor, Eiken Chemical Co. Ltd, Tokyo, Japan) to their home address, which included two sample collection tubes, instructions for use and a reply paid envelope. Participants collected samples from two different bowel motions at home. Dietary or medicine restrictions were not required. Participants were asked to post the collected samples to the Bowel Health Service laboratory within 2 weeks of collection of the first sample. Samples collected incorrectly were not analysed, and a new FIT kit was sent to the participant for the opportunity to repeat the test.

If FIT samples were not received by six weeks after date of the initial mail-out, a reminder letter was sent (Cole et al., 2003). People were excluded from further communications if they opted out from the study or indicated that they had either completed a FIT, or had colonoscopy in the previous 6 months. Participation was defined as return of a FIT kit within 12 weeks of invitation to screen.

\section{Blood test screening option for FIT non-participants}

People allocated to the intervention group were invited to a FIT-based screening program exactly as done with the control group (Figure 1). However FIT non-participants at 12 weeks were then sent a letter inviting them to screen using the methylated BCAT1/IKZF1 blood test (Clinical Genomics, Sydney, Australia) and instructed to call the study helpline to arrange a referral for phlebotomy. The phone call was recorded as the study outcome "interest in screening by blood test". These individuals were sent the referral, an information sheet specific for screening by blood testing and a blood test consent form. Phlebotomy was arranged through local blood collection centres (Healthscope Ltd, Adelaide South Australia) and blood was collected $(18 \mathrm{~mL}, \mathrm{~K} 3$-EDTA) without dietary or medicine restrictions. Six weeks after blood test offer, a reminder letter was sent to intervention group nonparticipants. Participation was defined as completion of a CRC screening test (either the blood test or the original FIT) within 12 weeks of the blood test screening offer. People who at any stage during the study who indicated that they were unsuitable to complete a FIT (either for personal or medical reasons, regardless of randomisation, Figure 1) were excluded from further FIT reminders and offers, and became part of a subgroup that was subsequently offered the blood test.

\section{Sample analysis}

Barcoded FIT specimen collection devices were analysed following return to the laboratory using an automated analyser according to manufacturer's instructions (OC-Sensor DIANA, Eiken Chemical Co. Ltd, Tokyo, Japan). A test was considered positive if haemoglobin $(\mathrm{Hb})$ concentration in either sample exceeded the test positivity threshold of $20 \mu \mathrm{g} \mathrm{Hb} / \mathrm{g}$ faeces 


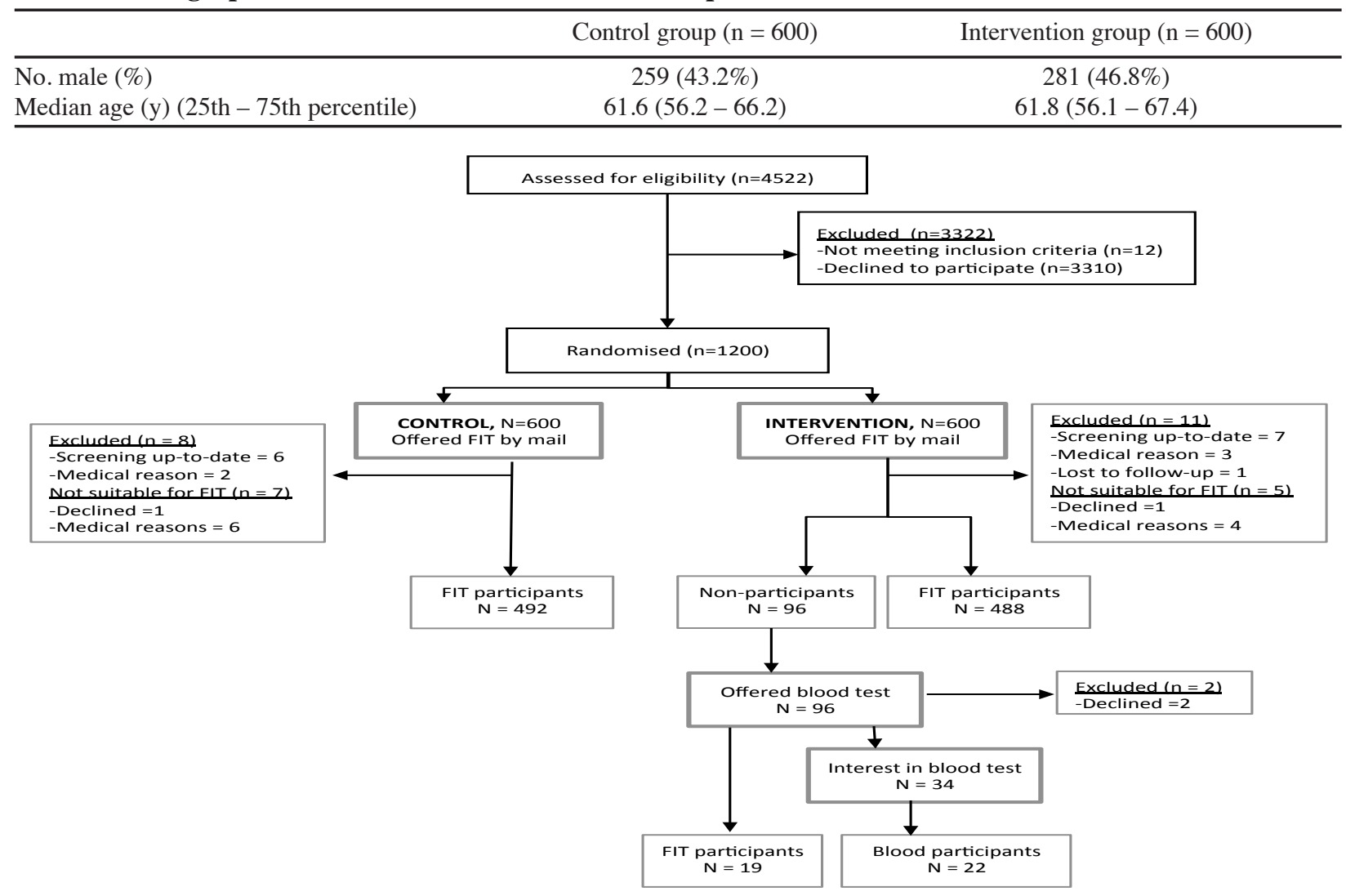

Figure 1. CONSORT Flow Diagram Showing Exclusions and Participation at Each Stage of the Study.

(100ng $\mathrm{Hb} / \mathrm{mL}$ buffer).

Blood samples were couriered at $4^{\circ} \mathrm{C}$ to a local laboratory (Flinders Centre for Innovation in Cancer, Bedford Park, South Australia) and plasma was separated from whole blood by centrifugation $(1500 \mathrm{~g}, 10 \mathrm{~min}$, room temperature, brakes disabled). Plasma specimens were stored at $-80^{\circ} \mathrm{C}$ and transported to Clinical Genomics for DNA extraction and assay of $B C A T 1 / I K Z F 1$ methylation. Briefly, silica-coated magnetic beads were used to isolate the cell free DNA from plasma. The DNA was bisulphite converted and re-purified before assayed for appearance of methylated $B C A T I$ and $I K Z F 1$ using real time quantitative multiplexed PCR. Assays were performed in triplicate. An internal control, beta-actin (ACTB1), was measured to confirm successful recovery of bisulphite converted DNA. Any BCAT1/IKZF1 cycle-threshold signal above background fluorescence level indicating the presence of hypermethylated DNA from either marker was considered a positive result (see reference (Pedersen et al., 2015b) for detailed methods).

Individuals who were positive with the FIT or blood test were recommended to have colonoscopy.

\section{Data analysis}

Primary outcome of screening participation between control and intervention groups was compared with a Chi-square test. Group characteristics were compared with Chi-square tests, a Mann-Whitney test, and a KruskalWallis one-way analysis of variance. Statistical analyses were performed with SigmaStat. A p-value less than 0.05 was considered statistically significant.

\section{Results}

Study population

There were 313 enrolled individuals from East Adelaide Healthcare, 521 from Chandlers Hill Surgery and 366 from Flagstaff Hill Medical Centre who were randomised to control or intervention. There were no differences in the demographics between the control and intervention groups (Table 1).

\section{Control group participation}

The enrolled numbers and participation are shown in the CONSORT diagram (Figure 1). Following offer of the standard FIT program to 600 people of the control group, $1.3 \%$ were excluded, $1.2 \%$ indicated they were unsuitable to complete FIT (with reasons indicated in Figure 1), and $82.0 \%$ participated with FIT. Median time between offer of FIT and return of the completed kit was 13 days (range: 4-77 days).

\section{Intervention group participation}

Following the original offer of the FIT to the intervention group, $1.8 \%$ were excluded, $0.8 \%$ indicated they were unsuitable to complete FIT (with reasons indicated in Figure 1), and $81.3 \%$ participated with FIT. After 12 weeks there were 96 people who were FIT nonparticipants who were offered the blood test for screening.

There were 34 people (35.4\%) that registered interest and $22(23 \%)$ completed the blood test. Median time for blood test uptake following offer was 12 days (range: 2-60 days). There were 19 people $(19.8 \%)$ that completed their 
original FIT after receiving the blood test offer. Overall participation in the FIT/blood intervention group (i.e. completion of either screening test) was $88 \%$, significantly greater than participation in the control group $(\mathrm{p}=0.003)$.

\section{Screening test uptake in those unsuitable for FIT}

Following the offer of the FIT, 12 invitees spread across both groups indicated that they were unsuitable for screening with FIT (10 due to benign bleeding conditions and 2 due to personal reasons), and all were offered to screen by the blood test. Ten requested a blood test referral, and all ten completed the test $(10 / 12,83 \%)$. Of the two subjects that did not take up the offer of the blood test, one subsequently indicated that they had undergone recent screening through another source.

Median time between blood test offer and blood collection in this subgroup was 4.5 days (range: $2-10$ days), which was significantly less than the time for FIT uptake in the control group and the time for blood test uptake in the intervention group $(\mathrm{p}<0.001)$.

\section{Discussion}

This study demonstrates that non-participants in a FIT-based CRC screening program will subsequently participate if offered a blood test for CRC. The combined effect of the FIT plus blood test phases of the program resulted in a significantly greater level of participation compared to that of a conventional FIT-only program even in this group of highly motivated study subjects where the initial FIT participation rate was high.

Surveys indicate a preference for blood sampling as a screening mode (Osborne et al., 2012; Benning et al., 2014), however this will not necessarily translate to actual test uptake, because collection location (home versus clinic) and cost are also important attributes determining test preference, with faecal sampling considered more convenient than attending a clinic for blood collection (Osborne et al., 2012). It is important to assess if a blood test can increase screening program participation. Prior to our research, only one study had assessed this in colonoscopy non-participants, where it was found that $82.6 \%$ of colonoscopy non-participants completed a blood test when offered the test as part of a face-to-face consultation with the primary care practitioner (Adler et al., 2014). The current study is the first study to assess blood test uptake in FIT-non-participants, which is the screening test used in many programs worldwide. We found that almost a third $(29.6 \%)$ of the FIT nonparticipants completed the blood test.

Convenience of blood draw has been previously shown to be a key factor in the decision to screen with a blood test (Adler et al., 2014) and this may be one reason for the difference in participation rates between our study and the other blood test participation study (Adler et al., 2014). Collection of blood within our study was limited to two hour weekday morning appointment, in comparison to the latter study where blood collection was performed during the visit to the General Practice.

Our study has shown that including a blood test option within a FIT-based CRC screening program is an effective way to increase overall screening participation. Not surprisingly, provision of the blood test was effective for the sub-population that indicated that they were unsuitable for FIT. Dislike of faecal sampling is known to be a common reason for refusal of screening with faecal sample based tests (Worthley et al., 2006), but it now seems likely that people exclude themselves, in part, from screening with FIT due to having known gastrointestinal conditions that result in rectal bleeding, potentially a false (for CRC) positive FIT result and unnecessary colonoscopy. This is a significant issue as almost $50 \%$ of people older than 50 years experience overt rectal bleeding from symptomatic haemorrhoids (Fox et al., 2014). Of 12 people who informed the study investigators that they were unsuitable for FIT, 10 completed the blood test within a significantly shorter median 4.5 days after receiving the offer compared to the median 13 days for FIT. The option of a CRC screening blood test improves the equity of screening, as people unsuitable for occult blood screening now have a viable and non-invasive alternative first-line test.

We conducted this initial study with participating General Practices using study volunteers because it was an ethically acceptable approach to explore the place of the new screening test. Sequential offers of FIT then blood test to FIT non-participants, also ensured invitees had the opportunity to participate using the contextually-relevant preferred test. However there are other program scenarios that might be feasible in the future, such as offering upfront the choice of faecal or blood sample based testing. Choice of test might be of interest in many international settings where general practitioners have a major role in primary care. Implementing CRC screening through primary care gives the opportunity for physicians to assess a person's suitability for test type, to advocate for screening and if blood, often with the ability to obtain a blood sample promptly on site.

Increasing screening test options to the general population may however create uncertainty as to whether screening is appropriate, and which screening modality is best for an individual (DeBourcy et al., 2008). Alternatively, more options could assist in improving participation by allowing individuals to choose a test which aligns with their preferences (Dam et al., 2013). Other studies have shown that sequential offers of different CRC screening options can improve overall participation rates (Hol et al., 2012; Senore et al., 2013). In developing a more acceptable test, specimen type and sampling mode, research with existing CRC screening tests indicates that preferred test attributes are non-invasive in nature, good accuracy, and minimal preparation requirements (Marshall et al., 2007). This is achievable with a blood test, particularly because more than $95 \%$ of screening age people (50-74y) are accustomed to blood testing for clinical purposes (Osborne et al., 2012). However, costs also need to be considered as a blood test has higher cost than FIT. A screening program based an initial offer of a relatively cheap occult blood test, followed by an offer of an alternative test type to those who cannot or will not participate might produce significant overall cost-benefit.

A limitation to the current study is that due to upfront consent requirements and an invitation process through 
Primary Care Practices with implied practice endorsement for screening, there was a very high participation with FIT, leaving very few FIT non-participants eligible for a blood test offer. We have previously shown that General Practice endorsement in the screening process significantly increases participation relative to no endorsement (Cole et al., 2002). Accordingly, FIT participation did not reflect the usual screening population participation rate in Australia which is less than $40 \%$ (AIHW, 2014). However, despite this being a study population biased towards FIT participation, we were still able to demonstrate increased screening activity by inclusion of a blood test offer within the FIT program.

A further limitation was that the intervention group received additional communications about screening compared to the control group. In addition, all invitees had originally been sent FIT kits that were still available and within their use-by date. As a consequence, 19 people were stimulated to return the previously neglected FIT kit. This additional increment plus the 22 who completed the blood test together resulted in a significantly higher program participation compared to the FIT only program and thus delivers an unexpected benefit.

In conclusion, the results from this study support the inclusion of a blood test option within a standard faecal based CRC screening program as an option for nonparticipants. It is now important to also apply this study design to a general population, in the same fashion as a national mail out, where screening participation rates are generally low. Further studies are also needed to determine best implementation modes and whether the additional screening option with the blood test can also promote continued screening within programs.

\section{Acknowledgements}

The authors would like to thank Dr Rohan Baker and David Murray for analysing all of the blood test samples, and Dawn Bastin for analysing all of the faecal immunochemical test samples.

\section{References}

Adler A, Geiger S, Keil A, et al (2014). Improving compliance to colorectal cancer screening using blood and stool based tests in patients refusing screening colonoscopy in Germany. BMC Gastroenterol, 14, 183.

AIHW 2014. National Bowel Cancer Screening Program monitoring report: 2012-13., Canberra, Australian Institute of Health and Welfare Canberra.

Benning TM, Dellaert BG, Dirksen CD, et al (2014). Preferences for potential innovations in non-invasive colorectal cancer screening: A labeled discrete choice experiment for a Dutch screening campaign. Acta Oncol, 53, 898-908.

Bresalier RS, Kopetz S, Brenner DE (2015). Blood-Based Tests for Colorectal Cancer Screening: Do They Threaten the Survival of the FIT Test? Dig Dis Sci, 60, 664-71.

Cole SR, Gregory T, Whibley A, et al (2012). Predictors of Re-participation in Faecal Occult Blood Test- Based Screening for Colorectal Cancer. Asian Pac J Cancer Prev, 13, 5989-94.

Cole SR, Tucker GR, Osborne JM, et al (2013). Shift to earlier stage at diagnosis as a consequence of the National Bowel
Cancer Screening Program. Med J Aust, 198, 327-30.

Cole SR, Young GP, Byrne D, et al (2002). Participation in screening for colorectal cancer based on a faecal occult blood test is improved by endorsement by the primary care practitioner. J Med Screen, 9, 147-52.

Cole SR, Young GP, Esterman A, et al (2003). A randomised trial of the impact of new faecal haemoglobin test technologies on population participation in screening for colorectal cancer. J Med Screen, 10, 117-22.

Cole SR, Zajac I, Gregory T, et al (2011). Psychosocial variables associated with colorectal cancer screening in South Australia. Int J Behav Med, 18, 302-9.

DeBourcy AC, Lichtenberger S, Felton S, et al (2008). Community-based preferences for stool cards versus colonoscopy in colorectal cancer screening. J Gen Intern Med, 23, 169-74.

deVos T, Tetzner R, Model F, et al (2009). Circulating methylated SEPT9 DNA in plasma is a biomarker for colorectal cancer. Clin Chem, 55, 1337-46.

Fox A, Tietze PH, Ramakrishnan K (2014). Anorectal conditions: hemorrhoids. FP Essent, 419, 11-9.

Greiner KA, James AS, Born W, et al (2005). Predictors of fecal occult blood test (FOBT) completion among low-income adults. Prev Med, 41, 676-84.

Hardcastle JD, Chamberlain JO, Robinson MH, et al (1996). Randomised controlled trial of faecal-occult-blood screening for colorectal cancer. Lancet, 348, 1472-7.

Hol L, Kuipers EJ, van Ballegooijen M, et al (2012). Uptake of faecal immunochemical test screening among nonparticipants in a flexible sigmoidoscopy screening programme. Int J Cancer, 130, 2096-102.

Mandel JS, Bond JH, Church TR, et al (1993). Reducing mortality from colorectal cancer by screening for fecal occult blood. Minnesota Colon Cancer Control Study. $N$ Engl $J$ Med, 328, 1365-71.

Marshall DA, Johnson FR, Phillips KA, et al (2007). Measuring patient preferences for colorectal cancer screening using a choice-format survey. Value Health, 10, 415-30.

Miles A, Rainbow S, von Wagner C (2011). Cancer fatalism and poor self-rated health mediate the association between socioeconomic status and uptake of colorectal cancer screening in England. Cancer Epidemiol Biomarkers Prev, 20, 2132-40.

Mitchell SM, Ross JP, Drew HR, et al (2014). A panel of genes methylated with high frequency in colorectal cancer. $B M C$ Cancer, 14.

Osborne J, Wilson C, Moore V, et al (2012). Sample preference for colorectal cancer screening tests: Blood or stool? OJPM, 2, 326-31.

Pedersen S, Symonds E, Baker R, et al (2015a). Evaluation of an assay for methylated $B C A T I$ and $I K Z F 1$ in plasma for detection of colorectal neoplasia. BMC Cancer, In press.

Pedersen SK, Baker RT, McEvoy A, et al (2015b). A two-gene blood test for methylated DNA sensitive for colorectal cancer. PLoS One, 10, 125041.

Senore C, Ederle A, Benazzato L, et al (2013). Offering people a choice for colorectal cancer screening. Gut, 62, 735-40.

Symonds E, Young G, Osborne J, et al (2015). Comparison of a methylated two-gene (BCAT1-IKZF1) blood test to FIT for detection of colorectal neoplasia. Gastroenterology, $148,746$.

van Dam L, Kuipers EJ, Steyerberg EW, et al (2013). The price of autonomy: should we offer individuals a choice of colorectal cancer screening strategies? Lancet Oncol, 14, 38-46.

Worthley DL, Cole SR, Esterman A, et al (2006). Screening for colorectal cancer by faecal occult blood test: why people choose to refuse. Intern Med J, 36, 607-10. 
Erin L Symonds et al

Zorzi M, Fedeli U, Schievano E, et al (2014). Impact on colorectal cancer mortality of screening programmes based on the faecal immunochemical test. Gut. 